\title{
Electrodeposición de Níquel Duro con Ondas de Corriente Pulsante Triangulares
}

\author{
Bibian A. Hoyos ${ }^{(1) *}$, Luisa F. Martínez ${ }^{(2)}$ y Álvaro F. Salazar ${ }^{(2)}$ \\ (1) Universidad Nacional de Colombia, Escuela de Procesos y Energía, Apdo. Aéreo 1027, \\ Medellín-Colombia (e-mail: bahoyos@unal.edu.co) \\ (2) Universidad Nacional de Colombia, Facultad de Minas, Of. M4-214, Carrera 80 No. 65-223, \\ Medellín-Colombia
}

* autor a quien debe ser dirigida la correspondencia

\begin{abstract}
Resumen
Se estudia el efecto de la frecuencia y la densidad de corriente sobre la dureza en electrodepósitos de níquel obtenidos mediante corrientes pulsantes triangulares y rectangulares y su comparación con los obtenidos empleando corriente directa. Las mediciones de dureza se realizaron según la norma ASTM E384. La descripción de la morfología de los depósitos se realizó mediante un microscopio de barrido electrónico (SEM) según las normas ASTM E112, E3 y E93. Los resultados experimentales muestran que a $4 \mathrm{~A} / \mathrm{dm}^{2}$ la dureza de los depósitos está influida por la forma de onda, produciéndose durezas en el orden rampa descendente $>$ rectangular $>$ rampa ascendente. Para las condiciones exploradas, la mayor dureza se obtiene con onda rectangular a $20 \mathrm{~A} / \mathrm{dm}^{2}$ y $60 \mathrm{~Hz}$. Para ésta onda de corriente, la dureza disminuye al aumentar el ciclo de trabajo. La dureza tiene un aumento de hasta un $300 \%$ cuando se emplea corriente pulsante frente a los valores alcanzados con corriente directa.
\end{abstract}

Palabras clave: corriente pulsante, electrodeposición de níquel, dureza, ondas triangulares

\section{Electroplating of Hard Nickel With Triangular Pulse Current Waveforms}

\begin{abstract}
In this paper it is studied the frequency and current density effect on hardness of nickel electroplating obtained by means of triangular and rectangular pulse current and their comparison with those obtained using direct current. Hardness measurements were done following the ASTM E384 standards. Morphologic qualitative description of deposits was done using a Scanning Electronic Microcopy (SEM) according with the ASTM E112, E3 and E93 standards. The experimental results showed that at $4 \mathrm{~A} / \mathrm{dm}^{2}$ the hardness of deposits is affected by the current waveform, producing hardness in the order: ramp-down > rectangular > ramp-up. For the experimental conditions explored here, the highest value of hardness was obtained at t $20 \mathrm{~A} / \mathrm{dm} 2$ and $60 \mathrm{~Hz}$. For this current waveform the hardness value diminishes when the duty cycle increases. The hardness increases up to $300 \%$ when using pulse current instead on direct current.
\end{abstract}

Keywords: pulse current, nickel electroplating, hardness, triangular waves 


\section{INTRODUCCIÓN}

Desde mediados del siglo pasado las industrias de depósitos por vía electrolítica o también llamadas industrias galvánicas, han tenido una gran expansión gracias a su capacidad para reducir las pérdidas de metales debidas a la corrosión y por el mejoramiento que se puede lograr de propiedades como la dureza y la resistencia a la abrasión.

Aunque la técnica del recubrimiento electrolítico se ha llevado a cabo durante muchos años mediante fuentes de corriente continua y con ésta se han logrado buenos resultados, desde hace algún tiempo se ha encontrado que usando ondas de corriente pulsante, el proceso puede ser mejorado (Devaraj y Seshadri, 1996, DiBari. 2002) y la electrodepositación pulsada ha ido ganando aceptación dentro de algunos sectores como el metalúrgico, automotriz, electrónico y aeronáutico, ya que mediante esta técnica se pueden obtener mejoras en la pureza y en las propiedades funcionales de los depósitos (Hansal et al., 2006; Youssef et al., 2004; Bioh y Ritzdorf, 2004 ; Georgianou y Veyret, 2002).

El valor de la dureza es una medida comúnmente usada para dar una indicación general de la fortaleza del material, así como de la resistencia a rayarse y desgastarse. Un depósito metálico con un tamaño de grano pequeño es, en general, más duro y mucho más resistente que un material que presente un tamaño de grano mayor.

En un estudio de comparación de la depositación de níquel en baños tipo watts sin aditivos empleando corriente pulsante y corriente continua (Devaraj y Seshadri, 1996), se encontró que empleando corriente pulsante con altas densidades de corriente pico y bajas frecuencias (entre $10 \mathrm{y}$ $100 \mathrm{~Hz}$ ) se producen depósitos con tamaño de grano más pequeño, con aumento en la dureza y disminución de la porosidad que los obtenidos empleando corriente continua. Otros trabajos han revelado que operando con ondas pulsantes de tiempos de encendido cortos, se producen depósitos superficiales lisos y con ausencia de poros (El-Sherik et al., 1996), mientras que operando con tiempos de encendido largos se produce un aumento en el número y tamaño de poros sobre la superficie de los depósitos.

También se ha mostrado (Wong et al., 1999) cualitativa y cuantitativamente que la forma de onda rectangular no produce la mejor superficie final en la electrodepositación pulsada de níquel, ya que esta forma de onda está relacionada con una alta densidad de corriente pico continua, lo cual facilita el crecimiento de los granos y disminuye la velocidad de formación de nuevos núcleos sobre la superficie del cátodo. En estudios más recientes (Wong et al., 2001 a, b), se encontró que el empleo de ondas de corriente en forma de rampa descendente o un aumento momentáneo en un escalón de corriente rectangular produce un depósito con una estructura de grano fino.

En este artículo se estudia el efecto de la frecuencia $(10$ y $60 \mathrm{~Hz})$ y la densidad de corriente (4 y 20 $\mathrm{A} / \mathrm{dm}^{2}$ ) sobre la dureza en los electrodepósitos de níquel obtenidos mediante corrientes pulsantes triangulares y rectangulares y su comparación con los obtenidos empleando corriente continua.

\section{PROCEDIMIENTO EXPERIMENTAL}

Los diferentes tipos de onda utilizados en este estudio se muestran en la figura 1. Adicionalmente, algunos de los parámetros que caracterizan a las ondas de corriente pulsante se listan en las ecuaciones 1 a 4 :

$\%$ Ciclo de Trabajo $=\frac{t_{C}}{t_{C}+t_{a}} * 100$

$$
f=\frac{1}{t_{c}+t_{a}}
$$


Para la onda de forma rectangular:

$i_{\text {prom }}=\frac{t_{C}}{t_{c}+t_{a}} * i_{c}$

Para las ondas en forma de rampas:

$i_{\text {prom }}=\frac{1}{2} * \frac{t_{C}}{t_{c}+t_{a}} * i_{c}$

en las cuales $f$ es la frecuencia del pulso, $i_{\text {prom }}$ la densidad de corriente promedio, $i_{c}$ la densidad de corriente pico catódica, $t_{a}$ es el tiempo de pausa o de apagado y $t_{c}$ el tiempo encendido o de duración de pulso.

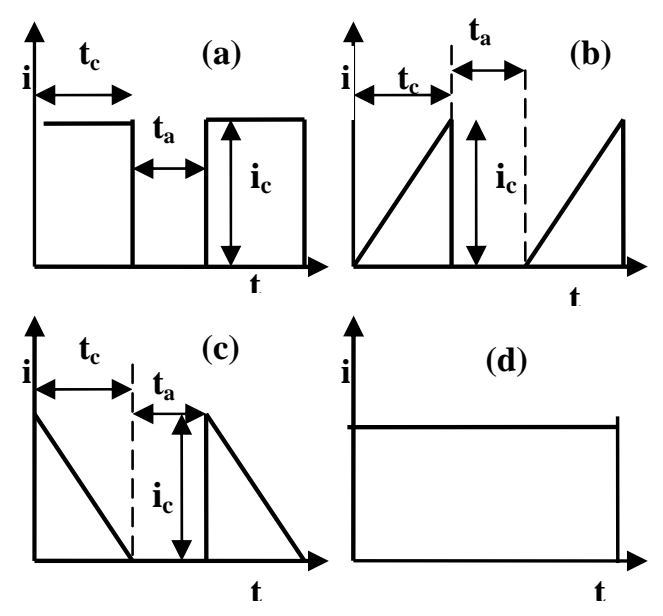

Fig. 1: Tipos de onda empleados: (a) rectangular; (b) rampa ascendente; (c) rampa descendente y (d) corriente directa.

En los experimentos de electrodepositación, la composición del baño fue: $312.5 \mathrm{~g} / \mathrm{l}$ de sulfato de níquel heptahidratado, $45 \mathrm{~g} / \mathrm{l}$ de cloruro de níquel hexahidratado y $37.5 \mathrm{~g} / \mathrm{l}$ de ácido bórico. El electrolito fue constantemente agitado por medio de cuatro agitadores mecánicos a $100 \mathrm{rpm}$, la temperatura se mantuvo constante en $55 \pm 1{ }^{\circ} \mathrm{C}$ y el $\mathrm{pH}$ inicial de la solución fue de 4.3 que es un valor típico usado en este tipo de recubrimientos (Schlesinger y Paunovic, 2000).

Se utilizaron como sustrato (cátodo) láminas cuadradas de cobre de $1 \mathrm{~mm}$ de espesor, con lados de $1.5 \mathrm{~cm}, 2.29 \mathrm{~cm}, 3.35 \mathrm{~cm}$ y $5.12 \mathrm{~cm}$. Las piezas fueron pulidas usando lijas de agua número $400 \mathrm{y}$ 600 , posteriormente se les realizó un pretratamiento para eliminar impurezas que disminuyen la adherencia del depósito de níquel. Las etapas del pretratamiento se muestran en la tabla 1. Entre cada una de las etapas mencionadas se colocaron enjuagues intermedios a fin de minimizar arrastres de reactivos de una etapa a la siguiente.

Tabla 1: Etapas para el pretratamiento del sustrato.

\begin{tabular}{|c|c|c|}
\hline Etapa & Condiciones de operación & Reactivos \\
\hline Desengrase químico & $70-90{ }^{\circ} \mathrm{C}, 7-10$ minutos & Desengrase alcalino \\
\hline Decapado & Temperatura ambiente & $\mathrm{H}_{2} \mathrm{SO}_{4} 50 \% \mathrm{~V}, \mathrm{HCl} 50 \% \mathrm{~V}$ \\
\hline Desengrase electrolítico & $60-70{ }^{\circ} \mathrm{C}, 4-10 \mathrm{~A} / \mathrm{dm}^{2}$ & Desengrase alcalino para \\
& $5-9$ Voltios, $3-5$ minutos & $\mathrm{HCl} 5 \% \mathrm{~V}$ \\
\hline Activado & Temperatura ambiente & \multicolumn{2}{|c|}{ uso elico } \\
\hline
\end{tabular}


Se fijó el espesor del depósito en $50 \mu \mathrm{m}$ y el ciclo de trabajo en 30\%, las pruebas experimentales se realizaron según las condiciones que se muestran en la tabla 2.

Tabla 2: Variables experimentales para los formas de onda de corriente (Ciclo de trabajo = 30\%).

\begin{tabular}{|c|l|l|l|l|l|l|l|l|l|l|l|l|l|l|}
\hline Tipo de onda & \multicolumn{4}{|c|}{ Rectangular } & \multicolumn{4}{c|}{ Rampa Ascendente } & \multicolumn{3}{c|}{ Rampa Descendente } & \multicolumn{3}{c|}{ Continua } \\
\hline $\mathrm{i}_{\text {prom }}\left(\mathrm{A} / \mathrm{dm}^{2}\right)$ & 4 & 4 & 20 & 20 & 4 & 4 & 20 & 20 & 4 & 4 & 20 & 20 & 4 & 20 \\
\hline$f(\mathrm{~Hz})$ & 10 & 60 & 10 & 60 & 10 & 60 & 10 & 60 & 10 & 60 & 10 & 60 & & \\
\hline
\end{tabular}

Las mediciones de dureza se realizaron mediante un microdurometro tipo $M$ (Shimadzu) con resolución de $0.5 \mu \mathrm{m}$, según la norma ASTM E384. A cada pieza se le realizaron cinco mediciones de microdureza alrededor de su centro con una carga de $50 \mathrm{gf}$ y se reporta el promedio de éstas mediciones. La obtención de las fotografías para la descripción cualitativa del tamaño de grano se hizo mediante un microscopio de electrónico de barrido (SEM) siguiendo los lineamientos de las normas ASTM E112, E3 y E93.

\section{RESULTADOS Y DISCUSIÓN}

La figura 2 muestra que el depósito con el valor más alto de dureza se consiguió empleando una onda rectangular, con densidad de corriente de $20 \mathrm{~A} / \mathrm{dm}^{2}$ y una frecuencia de $60 \mathrm{~Hz}$. Claramente se observa que el valor de la dureza tiene un aumento considerable de hasta un $300 \%$ cuando se emplea corriente pulsante frente a los valores alcanzados con corriente continua. Las variaciones de los valores de dureza con respecto a la densidad de corriente muestran que se obtienen depósitos más duros cuando se trabaja a densidades de corriente altas, para el presente caso $20 \mathrm{~A} / \mathrm{dm}^{2}$. Esto es debido a que corrientes altas aumentan la velocidad de nucleación y reducen la velocidad global de crecimiento de los cristales y por lo tanto se producen depósitos con una estructura de grano más fino que a su vez se refleja en un aumento de dureza.

Con una densidad de corriente de $4 \mathrm{~A} / \mathrm{dm}^{2}$ la dureza de los depósitos está influida por la forma de onda, produciéndose durezas en el orden: rampa descendente > rectangular > rampa ascendente. Esto es debido a que para la misma corriente promedio, la rampa descendente tiene la corriente pico más alta al comienzo de la depositación, lo que produce una mayor cantidad inicial de núcleos y por lo tanto mayor dureza. A $20 \mathrm{~A} / \mathrm{dm}^{2}$ la onda rectangular produce mayor dureza que la rampa descendente, debido posiblemente a la evolución de hidrogeno que puede aparecer con las altas corrientes pico requeridas para la rampa descendente en este caso. La generación de burbujas de hidrogeno produce un deposito poroso con una baja dureza. Para densidades de corriente promedio bajas $\left(4 \mathrm{~A} / \mathrm{dm}^{2}\right.$ ) se observa que siempre se obtienen valores de dureza más altos cuando se emplean frecuencias bajas $(10 \mathrm{~Hz})$. Con densidades de corriente de $20 \mathrm{~A} / \mathrm{dm}^{2}$, se obtiene en casi todos los casos mayor dureza a una frecuencia de $60 \mathrm{~Hz}$.

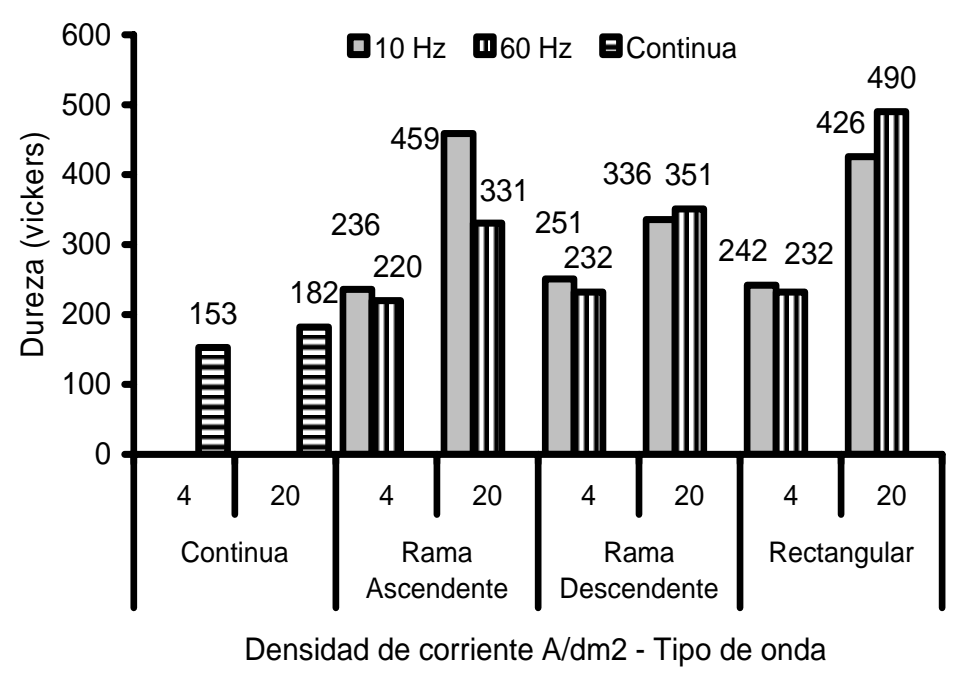

Fig. 2. Efecto de la frecuencia, densidad de corriente y el tipo de onda sobre el valor de la dureza (Ciclo de trabajo $=30 \%$ ). 
En la figura 3 se muestran los resultados obtenidos al variar el ciclo de trabajo para los experimentos realizados bajo las condiciones con las que se obtuvo el mayor valor de dureza: Onda rectangular a $20 \mathrm{~A} / \mathrm{dm}^{2}$. Se puede observar que el valor de la dureza disminuye al aumentar el ciclo de trabajo y que para un ciclo de trabajo de $70 \%$, se aumenta la dureza cuando se trabaja con bajas frecuencias, en contraposición a lo que sucede con ciclos de trabajo menores.

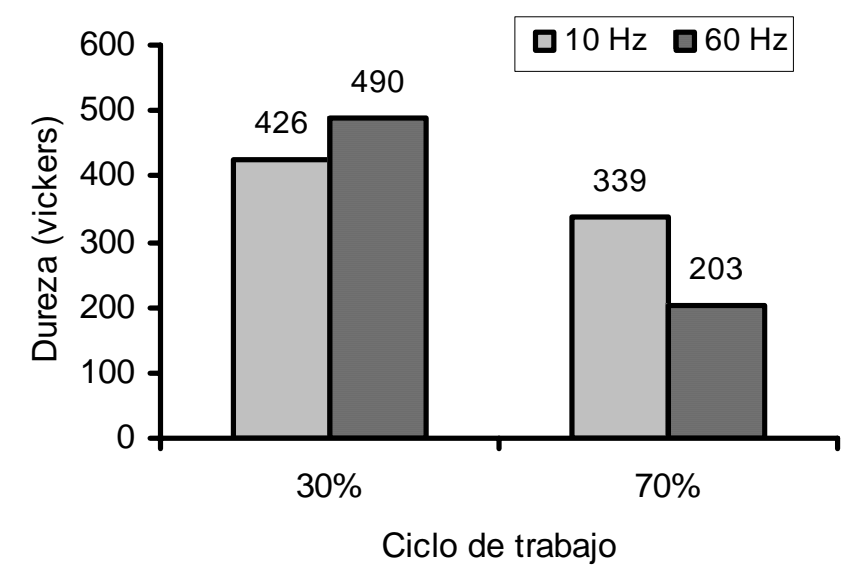

Fig. 3. Efecto del ciclo de trabajo sobre la dureza con onda rectangular a $20 \mathrm{~A} / \mathrm{dm}^{2}$.

El depósito obtenido con corriente continua, muestra una estructura rugosa con aglomerados en forma de cuña que en general presentaran menor adherencia con el sustrato (fig. 4a). La onda rectangular y la rampa ascendente (fig. 4 b y 4c) presentan granos diferenciables con algún grado de coalescencia entre ellos, lo que lleva a suponer que además de la nucleación, se produjo crecimiento de los núcleos cristalinos. La rampa descendente (fig. 4d) produce granos más pequeños que los otros tipos de ondas, con prácticamente ninguna coalescencia, lo que explica los mayores valores de dureza obtenidos con esta onda.

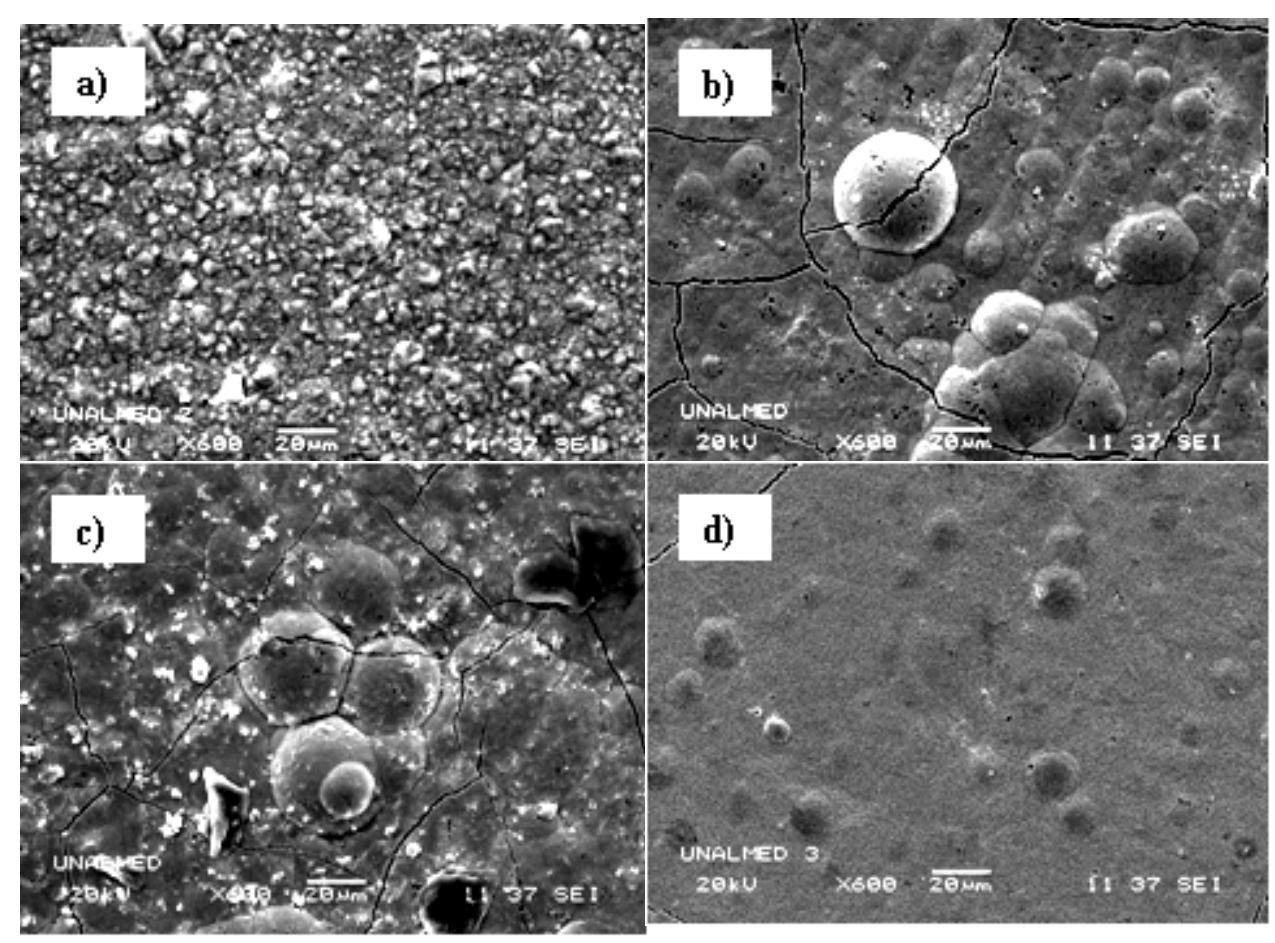

Fig. 4. Morfología de los depósitos obtenidos para las diferentes formas de onda a $4 \mathrm{~A} / \mathrm{dm}^{2}$ y $60 \mathrm{~Hz}$ : (a) corriente continua, (b) rectangular, (c) rampa ascendente y $(\mathrm{d})$ rampa descendente. 


\section{CONCLUSIONES}

En las condiciones de este estudio, el valor de la dureza de los depósitos se puede aumentar hasta un $300 \%$ cuando se emplea corriente pulsante en lugar de corriente continua en la obtención de los electrodepósitos.

De acuerdo a los experimentos realizados, fijando espesor del depósito y porcentaje de ciclo de trabajo, el mayor valor de dureza se obtiene empleando onda rectangular a $20 \mathrm{~A} / \mathrm{dm}^{2}$ y $60 \mathrm{~Hz}$.

No se conserva una misma tendencia cuando se varían las densidades de corriente, ya que a 4 $\mathrm{A} / \mathrm{dm}^{2}$ se obtiene el mayor valor de dureza cuando se opera con rampa descendente, contrario a lo que sucede a $20 \mathrm{~A} / \mathrm{dm}^{2}$, con la que la onda rectangular produce los mayores valores de dureza.

Para la onda de corriente rectangular, se obtienen electro-depósitos más duros operando a ciclos de trabajo de $30 \%$ con frecuencia de $60 \mathrm{~Hz}$.

\section{AGRADECIMIENTOS}

Este trabajo se pudo llevar a cabo gracias a la financiación de COLCIENCIAS (código del proyecto: 1118-08-17212) y de la dirección de investigación de la Universidad Nacional de Colombia - Sede Medellín (DIME).

\section{REFERENCIAS}

Bioh, K. y T. Ritzdorf; Electrical Waveform Mediated Throgh-Mask Deposition of Solder Bumps for Wafer Level Packaging, J. Electrochem. Soc.: 151 (5), C342-C347 (2004).

Devaraj, G. y S.K. Seshadri; Pulsed electrodeposition of nickel. Plating and Surface Finishing: agosto, 6266 (1996).

Dibari, G.A: Chronology of Nickel Electroplating, Metal Finishing: 100, 34-48 (2002).

El-Sherik, A.M., U. Erb y J. Page; Microstructural Evolution in Pulse Plated Nickel Electrodeposits, Surface and Coatings Technology: 88, 70-78 (1996).

Georgiadou M. y D. Veyret; Modeling of Transient Electrochemical Systems Involving Moving Boundaries, J. Electrochem. Soc.: 149 (6), C324-C330 (2002).

Hansal, W., y otros cuatro autores; Pulse reverse plating of Ni-Co alloys: Depotion Kinetics of Watts, Sulfamate and choride electrolytes, Electrochim. Acta: 52 (3), 1145-1151 (2006).

Schlesinger, M y M. Paunovic; Modern Electroplating, $4^{\text {th }}$ Ed, 420-422, John Wiley \& Sons, New York, U. S. A. (2000)

Wong, K.P., K.C. Chan y T.M. Yue; A study of surface finishing in pulse current electroforming of nickel by utilizing different shaped waveforms, Surface and Coatings Technology: 115, 132-139 (1999).

Wong, K.P., K.C. Chan y T.M. Yue; Influence of spike current in different shaped waveforms on the surface finish of nickel electroforms. Surface and Coatings Technology: 140, 284-292, (2001a).

Wong, K.P., K.C. Chan y T.M. Yue; $A$ study of hardness and grain size in pulse current electroforming of nickel using different shaped waveforms, J. Appl. Electrochem.: 31, 25-34 (2001b).

Youssef Kh., C.C. Koch y P.S. Fedkiw; Influence of Additives and Pulse Electodeposition Parameters on Production of Nanocrystalline Zinc for Zinc Coloride, J. Electrochem. Soc.: 151(2), C103-C111 (2004). 